\title{
Best proximity point results in geodesic metric spaces
}

\author{
Maryam A Alghamdi ${ }^{1}$, Mohammed A Alghamdi ${ }^{2}$ and Naseer Shahzad ${ }^{2 *}$
}

${ }^{\text {*Correspondence: }}$

nshahzad@kau.edu.sa

${ }^{2}$ Department of Mathematics, King Abdulaziz University, P.O. Box 80203

Jeddah, 21859, Saudi Arabia

Full list of author information is

available at the end of the article

\begin{abstract}
In this paper, the existence of a best proximity point for relatively $u$-continuous mappings is proved in geodesic metric spaces. As an application, we discuss the existence of common best proximity points for a family of not necessarily commuting relatively $u$-continuous mappings.
\end{abstract}

\section{Introduction}

Let $A$ be a nonempty subset of a metric space $(X, d)$ and $T: A \rightarrow X$. A solution to the equation $T x=x$ is called a fixed point of $T$. It is obvious that the condition $T(A) \cap A \neq \emptyset$ is necessary for the existence of a fixed point for $T$. But there occur situations in which $d(x, T x)>0$ for all $x \in A$. In such a situation, it is natural to find a point $x \in A$ such that $x$ is closest to $T x$ in some sense. The following well-known best approximation theorem, due to Ky Fan [1], explores the existence of an approximate solution to the equation $T x=x$.

Theorem 1 [1] Let $A$ be a nonempty compact convex subset of a normed linear space $X$ and $T: A \rightarrow X$ be a continuous function. Then there exists $x \in A$ such that $\|x-T x\|=$ $\operatorname{dist}(T x, A)=\inf \{\|T x-a\|: a \in A\}$.

The point $x \in A$ in Theorem 1 is called a best approximant of $T$ in $A$. Let $A, B$ be nonempty subsets of a metric space $X$ and $T: A \rightarrow B$. A point $x_{0} \in A$ is called a best proximity point of $T$ if $d\left(x_{0}, T x_{0}\right)=\operatorname{dist}(A, B)$. Some interesting results in approximation theory can be found in [2-8].

Eldred et al. [2] defined relatively nonexpansive mappings and used the proximal normal structure to prove the existence of best proximity points for such mappings.

Definition 2 [2] Let $A, B$ be nonempty subsets of a metric space $(X, d)$. A mapping $T$ : $A \cup B \rightarrow A \cup B$ is said to be a relatively nonexpansive mapping if

(i) $T(A) \subseteq B, T(B) \subseteq A$;

(ii) $d(T x, T y) \leq d(x, y)$, for all $x \in A, y \in B$.

Theorem 3 [2] Let $(A, B)$ be a nonempty, weakly compact convex pair in a Banach space X. Let $T: A \cup B \rightarrow A \cup B$ be a relatively nonexpansive mapping and suppose $(A, B)$ has a proximal normal structure. Then there exists $(x, y) \in A \times B$ such that

$$
\|x-T x\|=\|T y-y\|=\operatorname{dist}(A, B) .
$$

C 2012 Alghamdi et al.; licensee Springer. This is an Open Access article distributed under the terms of the Creative Commons Attribution License (http://creativecommons.org/licenses/by/2.0), which permits unrestricted use, distribution, and reproduction in any medium, provided the original work is properly cited. 
Remark 4 [2] Note that every nonexpansive self-map is a relatively nonexpansive map. Also, a relatively nonexpansive mapping need not be continuous.

In [8], Sankar Raj and Veeramani used a convergence theorem to prove the existence of best proximity points for relatively nonexpansive mappings in strictly convex Banach spaces.

Recently, Elderd, Sankar Raj and Veeramani [9] introduced a class of relatively $u$ continuous mappings and investigated the existence of best proximity points for such mappings in strictly convex Banach spaces.

Definition 5 [9] Let $A, B$ be nonempty subsets of a metric space $X$. A mapping $T: A \cup B \rightarrow$ $A \cup B$ is said to be a relatively $u$-continuous mapping if it satisfies:

(i) $T(A) \subseteq B, T(B) \subseteq A$;

(ii) for each $\varepsilon>0$, there exists a $\delta>0$ such that $d(T x, T y)<\varepsilon+\operatorname{dist}(A, B)$, whenever $d(x, y)<\delta+\operatorname{dist}(A, B)$, for all $x \in A, y \in B$.

Theorem 6 [9] Let A, B be nonempty compact convex subsets of a strictly convex Banach space $X$ and $T: A \cup B \rightarrow A \cup B$ be a relatively u-continuous mapping. Then there exists $(x, y) \in A \times B$ such that

$$
\|x-T x\|=\|y-T y\|=\operatorname{dist}(A, B)
$$

Remark 7 [9] Every relatively nonexpansive mapping is a relatively $u$-continuous mapping, but the converse is not true.

Example 8 [9] Let $\left(X=\mathbb{R}^{2},\|\cdot\|_{2}\right)$ and consider $A=\{(0, t): 0 \leq t \leq 1\}$ and $B=\{(1, s): 0 \leq$ $s \leq 1\}$. Define $T: A \cup B \rightarrow A \cup B$ by

$$
T(x, y)= \begin{cases}(1, \sqrt{y}) & \text { if } x=0 \\ (0, \sqrt{y}) & \text { if } x=1 .\end{cases}
$$

Then $T$ is relatively $u$-continuous, but not relatively nonexpansive.

Also, in [9], the authors proved the existence of common best proximity points for a family of commuting relatively $u$-continuous mappings.

The aim of this paper is to discuss the existence of a best proximity point for relatively $u$-continuous mappings in the frameworks of geodesic metric spaces. As an application, we investigate the existence of common best proximity points for a family of not necessarily commuting relatively $u$-continuous mappings.

\section{Preliminaries}

In this section, we give some preliminaries.

Definition 9 [10] A metric space $(X, d)$ is said to be a geodesic space if every two points $x$ and $y$ of $X$ are joined by a geodesic, i.e., a map $c:[0, l] \subseteq \mathbb{R} \rightarrow X$ such that $c(0)=x, c(l)=y$, and $d\left(c(t), c\left(t^{\prime}\right)\right)=\left|t-t^{\prime}\right|$ for all $t, t^{\prime} \in[0, l]$. Moreover, $X$ is called uniquely geodesic if there is exactly one geodesic joining $x$ and $y$ for each $x, y \in X$. 
The midpoint $m$ between two points $x$ and $y$ in a uniquely geodesic metric space has the property $d(x, m)=d(y, m)=\frac{1}{2} d(x, y)$. A trivial example of a geodesic space is a Banach space with usual segments as geodesic segments.

A point $z \in X$ belongs to the geodesic segment $[x, y]$ if and only if there exists $t \in[0,1]$ such that $d(z, x)=t d(x, y)$ and $d(z, y)=(1-t) d(x, y)$. Hence, we write $z=(1-t) x+t y$.

A subset $A$ of a geodesic metric space $X$ is said to be convex if it contains any geodesic segment that joins each pair of points of $A$.

The metric $d: X \times X \rightarrow \mathbb{R}$ in a geodesic space $(X, d)$ is convex if

$$
d(z,(1-t) x+t y) \leq(1-t) d(z, x)+t d(z, y)
$$

for any $x, y, z \in X$ and $t \in[0,1]$.

Definition 10 [10] A geodesic metric space $X$ is said to be strictly convex if for every $r>0$, $a, x$ and $y \in X$ with $d(x, a) \leq r, d(y, a) \leq r$ and $x \neq y$, it is the case that $d(a, p)<r$, where $p$ is any point between $x$ and $y$ such that $p \neq x$ and $p \neq y$, i.e., $p$ is any point in the interior of a geodesic segment that joins $x$ and $y$.

Remark 11 [10] Every strictly convex metric space is uniquely geodesic.

In [10], Fernández-León proved the existence and uniqueness of best proximity points in strictly convex metric spaces. For more details about geodesic spaces, one may check [11-13].

In the particular framework of geodesic metric spaces, the concept of global nonpositive curvature (global NPC spaces), also known as the CAT(0) spaces, is defined in [13] as follows.

Definition 12 A global NPC space is a complete metric space $(X, d)$ for which the following inequality holds true: for each pair of points $x_{0}, x_{1} \in X$ there exists a point $y \in X$ such that for all points $z \in X$,

$$
d^{2}(z, y) \leq \frac{1}{2} d^{2}\left(z, x_{0}\right)+\frac{1}{2} d^{2}\left(z, x_{1}\right)-\frac{1}{4} d^{2}\left(x_{0}, x_{1}\right) .
$$

Proposition 13 [13] If $(X, d)$ is a global NPC space, then it is a geodesic space. Moreover, for any pair of points $x_{0}, x_{1} \in X$ there exists a unique geodesic $\gamma:[0,1] \rightarrow X$ connecting them. For $t \in[0,1]$ the intermediate points $\gamma_{t}$ depend continuously on the endpoints $x_{0}, x_{1}$. Finally, for any $z \in X$,

$$
d^{2}\left(z, \gamma_{t}\right) \leq(1-t) d^{2}\left(z, x_{0}\right)+t d^{2}\left(z, x_{1}\right)-t(1-t) d^{2}\left(x_{0}, x_{1}\right) .
$$

Corollary 14 [13] Let $(X, d)$ be a global NPC space, $\gamma, \eta:[0,1] \rightarrow X$ be geodesics and $t \in$ $[0,1]$. Then

$$
d^{2}\left(\gamma_{t}, \eta_{t}\right) \leq(1-t) d^{2}\left(\gamma_{0}, \eta_{0}\right)+t d^{2}\left(\gamma_{1}, \eta_{1}\right)-t(1-t)\left[d\left(\gamma_{0}, \gamma_{1}\right)-d\left(\eta_{0}, \eta_{1}\right)\right]^{2}
$$

and

$$
d\left(\gamma_{t}, \eta_{t}\right) \leq(1-t) d\left(\gamma_{0}, \eta_{0}\right)+t d\left(\gamma_{1}, \eta_{1}\right)
$$


Corollary 14 shows that the distance function $(x, y) \longmapsto d(x, y)$ in a global NPC space is convex with respect to both variables. Consequently, all balls in a global NPC space are convex.

Example 15 Every Hilbert space is a global NPC space.

Example 16 Every metric tree is a global NPC space.

Example 17 A Riemannian manifold is a global NPC space if and only if it is complete, simply connected, and of nonpositive curvature.

More details about global NPC spaces can be found in [13-16].

We need the following notations in the sequel. Let $(X, d)$ be a metric space and $A, B$ be nonempty subsets of $X$. Define

$$
\begin{aligned}
& \operatorname{dist}(A, B)=\inf \{d(a, b): a \in A, b \in B\}, \\
& A_{0}=\{x \in A: d(x, y)=\operatorname{dist}(A, B), \text { for some } y \in B\}, \\
& B_{0}=\{y \in B: d(x, y)=\operatorname{dist}(A, B), \text { for some } x \in A\} .
\end{aligned}
$$

Given $C$ a nonempty subset of $X$, the metric projection $P_{C}: X \rightarrow 2^{C}$ is the mapping

$$
P_{C}(x)=\{y \in C: d(x, y)=\operatorname{dist}(x, C)\}, \quad \text { for every } x \in X,
$$

where $2^{C}$ denotes the set of all subsets of $C$.

Definition 18 [9] Let $A, B$ be nonempty convex subsets of a geodesic metric space. A mapping $T: A \cup B \rightarrow A \cup B$ is said to be affine if

$$
T(\lambda x+(1-\lambda) y)=\lambda T x+(1-\lambda) T y,
$$

for all $x, y \in A$ or $x, y \in B$ and $\lambda \in(0,1)$.

Definition 19 [8] Let $X$ be a metric space. A subset $C$ of $X$ is called approximatively compact if for any $y \in X$ and for any sequence $\left\{x_{n}\right\}$ in $C$ such that $d\left(x_{n}, y\right) \rightarrow \operatorname{dist}(y, C)$ as $n \rightarrow \infty,\left\{x_{n}\right\}$ has a subsequence which converges to a point in $C$.

In [13], Sturm presented the following result which ensures the existence and uniqueness of the metric projection on a global NPC space.

\section{Proposition 20}

(i) For each closed convex set $C$ in a global NPC space $(X, d)$, there exists a unique map $P_{C}: X \rightarrow C$ (projection onto $C$ ) such that

$$
d\left(P_{C}(x), x\right)=\inf _{y \in C} d(x, y) \quad \text { for every } x \in X
$$


(ii) $P_{C}$ is orthogonal in the sense that

$$
d^{2}(x, y) \geq d^{2}\left(x, P_{C}(x)\right)+d^{2}\left(P_{C}(x), y\right)
$$

for every $x \in X, y \in C$;

(iii) $P_{C}$ is nonexpansive,

$$
d\left(P_{C}(x), P_{C}(z)\right) \leq d(x, z) \quad \text { for every } x, z \in X
$$

Remark 21 Note that the existence of a unique metric projection does not need the compactness of $C$.

Remark 22 [13] For any subset $A$ of a global NPC space $(X, d)$, there exists a unique smallest convex set $c o(A)=\bigcup_{n=0}^{\infty} A_{n}$, containing $A$ and called convex hull of $A$. Where $A_{0}=A$, and for $n \in \mathbb{N}$, the set $A_{n}$ consists of all points in global NPC space $X$ which lie on geodesics which start and end in $A_{n-1}$.

Based on Proposition 20, Niculescu and Roventa [17] proved the Schauder fixed point theorem in the setting of a global NPC space.

Theorem 23 Let $C$ be a closed convex subset of a global NPC space $X$ with the property that the closed convex hull of every finite subset of $X$ is compact. Then every continuous map $T: C \rightarrow C$, whose image $T(C)$ is relatively compact, has a fixed point.

\section{Main results}

In this section, we will prove the existence of best proximity points for a relatively $u$-continuous mapping. Also, we obtain a result on the existence of common best proximity points for a family of not necessarily commuting relatively $u$-continuous mappings.

Proposition 24 Let $A, B$ be nonempty subsets of a metric space $X$ with $A_{0} \neq \emptyset$ and $T$ : $A \cup B \rightarrow A \cup B$ be a relatively $u$-continuous mapping. Then $T\left(A_{0}\right) \subseteq B_{0}$ and $T\left(B_{0}\right) \subseteq A_{0}$.

Proof Choose $x \in A_{0}$, then there exists $y \in B$ such that $d(x, y)=\operatorname{dist}(A, B)$. But $T$ is a relatively $u$-continuous mapping, then for each $\varepsilon>0$, there exists a $\delta>0$ such that

$$
d(p, q)<\delta+\operatorname{dist}(A, B) \quad \text { implies } \quad d(T p, T q)<\varepsilon+\operatorname{dist}(A, B)
$$

for each $p \in A, q \in B$. Since $d(x, y)<\delta+\operatorname{dist}(A, B)$ for any $\delta>0$, hence

$$
\operatorname{dist}(A, B) \leq d(T x, T y)<\varepsilon+\operatorname{dist}(A, B)
$$

for each $\varepsilon>0$. Therefore, $d(T x, T y)=\operatorname{dist}(A, B)$ and then $T(x) \in B_{0}$. This shows that $T\left(A_{0}\right) \subseteq B_{0}$. Similarly, it can be seen that $T\left(B_{0}\right) \subseteq A_{0}$.

Proposition 25 Let $A, B$ be nonempty closed convex subsets of a global NPC space $X$ with $A_{0} \neq \emptyset, T: A \cup B \rightarrow A \cup B$ be a relatively u-continuous mapping, and $P: A \cup B \rightarrow A \cup B$ be 
a mapping defined by

$$
P(x)= \begin{cases}P_{B}(x), & \text { if } x \in A, \\ P_{A}(x), & \text { if } x \in B .\end{cases}
$$

Then $T P(x)=P(T x)$ for all $x \in A_{0} \cup B_{0}$, i.e., $P_{A}(T(x))=T\left(P_{B}(x)\right)$ for $x \in A_{0}$ and $T\left(P_{A}(y)\right)=$ $P_{B}(T(y))$ for $y \in B_{0}$.

Proof Choose $x \in A_{0}$, then there exists $y \in B$ such that $d(x, y)=\operatorname{dist}(A, B)$. According to Proposition 20, since the metric projection is unique, we have $y=P_{B}(x)$ and $x=P_{A}(y)$. Recalling that $T$ is relatively $u$-continuous, therefore, as in the proof of Proposition 24, $d(T x, T y)=\operatorname{dist}(A, B)$. Thus, it follows that $T(x) \in B_{0}$ and $T(y) \in A_{0}$. Again, in view of the uniqueness of the projection operator, we have

$$
P_{A}(T(x))=T(y)=T\left(P_{B}(x)\right) .
$$

So, $P_{A}(T(x))=T\left(P_{B}(x)\right)$ for any $x \in A_{0}$. Similarly, it can be shown that $T\left(P_{A}(y)\right)=P_{B}(T(y))$ for any $y \in B_{0}$.

By an analogous argument to the proof of Theorem 3.1 [8], we can prove the following theorem.

Theorem 26 Let $A, B$ be two nonempty subsets of a global NPC space $X$ such that $A$ is closed convex and $B$ is closed. If $A_{0}$ is approximatively compact and $\left\{x_{n}\right\}$ is a sequence in $A_{0}$, and $y \in B$ such that $d\left(x_{n}, y\right) \rightarrow \operatorname{dist}(A, B)$, then $x_{n} \rightarrow P_{A}(y)$.

Proof Assume the contrary, then there exists $\varepsilon>0$ and a subsequence $\left\{x_{n_{m}}\right\}$ of $\left\{x_{n}\right\}$ such that

$$
d\left(x_{n_{m}}, y\right) \rightarrow \operatorname{dist}(A, B) \quad \text { but } \quad d\left(x_{n_{m}}, P_{A}(y)\right) \geq \varepsilon
$$

Since $A_{0}$ is approximatively compact, there exists a subsequence $\left\{x_{n_{m}^{\prime}}\right\}$ of $\left\{x_{n_{m}}\right\}$ which converges to a point $x \in A$. Hence,

$$
d\left(x_{n_{m}^{\prime}}, y\right) \rightarrow d(x, y)
$$

Also,

$$
d\left(x_{n_{m}^{\prime}}, y\right) \rightarrow \operatorname{dist}(A, B)
$$

Thus, $d(x, y)=\operatorname{dist}(A, B)$. By Proposition 20, it follows that $x=P_{A}(y)$. Finally, we obtain

$$
d\left(x_{n_{m}^{\prime}}, P_{A}(y)\right) \rightarrow d\left(x, P_{A}(y)\right) \geq \varepsilon,
$$

which implies that $x \neq P_{A}(y)$. This leads to a contradiction and therefore $x_{n} \rightarrow P_{A}(y)$. 
The following theorem guarantees the existence of best proximity points for a relatively $u$-continuous mapping in a global NPC space.

Theorem 27 Let A, B be nonempty closed convex subsets of a global NPC space $X$ with the property that the closed convex hull of every finite subset of $X$ is compact. Let $A_{0}, B_{0}$ be nonempty compact convex and $T: A \cup B \rightarrow A \cup B$ be a relatively u-continuous mapping. Then there exist $x_{0} \in A, y_{0} \in B$ such that

$$
d\left(x_{0}, T x_{0}\right)=d\left(y_{0}, T y_{0}\right)=\operatorname{dist}(A, B) .
$$

Proof By Proposition 24, since $T$ is a relatively $u$-continuous mapping, we have $T\left(A_{0}\right) \subseteq$ $B_{0}$ and $T\left(B_{0}\right) \subseteq A_{0}$. The result follows from Theorem 23 once we show that $P_{A} \circ T: A_{0} \rightarrow$ $A_{0}$ is a continuous mapping, where $P_{A}: X \rightarrow A$ is a metric projection operator.

To prove this, first notice that $P_{A}\left(B_{0}\right) \subseteq A_{0}$. Since $X$ is a global NPC space, by Proposition 20, we obtain that $P_{A}: X \rightarrow A$ is a continuous mapping. In what follows, we see that the mapping $T$ is continuous on $A_{0}$, In fact, let $\left\{x_{n}\right\}$ be a sequence in $A_{0}$ such that $x_{n} \rightarrow x_{0}$ for some $x_{0} \in A_{0}$. From Proposition 25, we have

$$
P_{B}\left(P_{A}\left(T x_{0}\right)\right)=P_{B}\left(T\left(P_{B} x_{0}\right)\right)=T\left(P_{A}\left(P_{B} x_{0}\right)\right)=T x_{0} .
$$

Notice that

$$
\begin{aligned}
d\left(x_{n}, P_{B}\left(x_{0}\right)\right) & \leq d\left(x_{n}, x_{0}\right)+d\left(x_{0}, P_{B}\left(x_{0}\right)\right) \\
& =d\left(x_{n}, x_{0}\right)+\operatorname{dist}(A, B) \rightarrow \operatorname{dist}(A, B)
\end{aligned}
$$

as $n \rightarrow \infty$. Since $T$ is relatively $u$-continuous, for each $\varepsilon>0$, there exists a $\delta>0$ such that $d(x, y)<\delta+\operatorname{dist}(A, B)$ implies $d(T x, T y)<\varepsilon+\operatorname{dist}(A, B)$ for all $x \in A, y \in B$. From (3.1), with this $\delta>0$, it follows that there is $N \in \mathbb{N}$ such that $d\left(x_{n}, P_{B}\left(x_{0}\right)\right)<\delta+\operatorname{dist}(A, B)$ for all $n \geq N$. This implies

$$
d\left(T\left(x_{n}\right), T\left(P_{B}\left(x_{0}\right)\right)\right)<\varepsilon+\operatorname{dist}(A, B)
$$

for all $n \geq N$. Therefore,

$$
d\left(T\left(x_{n}\right), P_{A}\left(T x_{0}\right)\right)=d\left(T\left(x_{n}\right), T\left(P_{B}\left(x_{0}\right)\right)\right) \rightarrow \operatorname{dist}(A, B) .
$$

This together with Theorem 26 implies that $T x_{n} \rightarrow P_{B}\left(P_{A}\left(T x_{0}\right)\right)=T x_{0}$. Thus, $T$ is continuous on $A_{0}$.

Now, since $P_{A} \circ T$ is a continuous mapping of $A_{0}$, by the Schauder fixed point theorem for a global NPC space, Theorem $23, P_{A} \circ T$ has a fixed point $x_{0} \in A_{0}$. From $P_{A}\left(T x_{0}\right)=x_{0}$, we find that $d\left(x_{0}, T x_{0}\right)=\operatorname{dist}\left(T x_{0}, A\right)$. But since $T x_{0} \in B_{0}$, there is $x^{\prime} \in A_{0}$ such that $d\left(x^{\prime}, T x_{0}\right)=$ $\operatorname{dist}(A, B)$. Consequently,

$$
\operatorname{dist}(A, B) \leq \operatorname{dist}\left(T x_{0}, A\right) \leq d\left(T x_{0}, x^{\prime}\right)=\operatorname{dist}(A, B),
$$


which gives

$$
\operatorname{dist}\left(T x_{0}, A\right)=\operatorname{dist}(A, B) .
$$

Thus, $d\left(x_{0}, T x_{0}\right)=\operatorname{dist}(A, B)$. This completes the proof.

Next, we will show that Theorem 27 is also true for an appropriate family of relatively $u$-continuous mappings. The following notations define the set of all best proximity points of a relatively $u$-continuous mapping:

$$
\begin{aligned}
& F_{A}(T)=\{x \in A: d(x, T x)=\operatorname{dist}(A, B)\}, \\
& F_{B}(T)=\{y \in B: d(y, T y)=\operatorname{dist}(A, B)\} .
\end{aligned}
$$

Theorem 28 Let A, B be nonempty closed convex subsets of a global NPC space $X$ with the property that the closed convex hull of every finite subset of $X$ is compact. Let $A_{0}, B_{0}$ be nonempty compact convex and $T: A \cup B \rightarrow A \cup B$ be a relatively u-continuous mapping. Let $T$ be affine. Then $F_{A}(T)$ is a nonempty compact convex subset of $A_{0}$ and $F_{B}(T)$ is a nonempty compact convex subset of $B_{0}$.

Proof It is obvious that $F_{A}(T)$ is a nonempty subset of $A_{0}$ by Theorem 27. Assume that $\left\{x_{n}\right\}$ is a sequence in $F_{A}(T)$ such that $x_{n} \rightarrow x_{0}$ for some $x_{0} \in A_{0}$. By the continuity of $T$ on $A_{0}$, we have $x_{0} \in F_{A}(T)$. Therefore, $F_{A}(T)$ is closed and then compact. Now we claim that $F_{A}(T)$ is convex. In fact, let $\lambda \in[0,1], x_{1}, x_{2} \in F_{A}(T)$, and $z=(1-\lambda) x_{1}+\lambda x_{2}$. Since the distance function $d$ is convex with respect to both variables, by Corollary 14, we have

$$
\begin{aligned}
\operatorname{dist}(A, B) & \leq d(z, T z) \\
& =d\left((1-\lambda) x_{1}+\lambda x_{2}, T\left((1-\lambda) x_{1}+\lambda x_{2}\right)\right) \\
& =d\left((1-\lambda) x_{1}+\lambda x_{2},(1-\lambda) T x_{1}+\lambda T x_{2}\right) \\
& \leq(1-\lambda) d\left(x_{1}, T x_{1}\right)+\lambda d\left(x_{2}, T x_{1}\right) \\
& =\operatorname{dist}(A, B) .
\end{aligned}
$$

This implies that $d(z, T z)=\operatorname{dist}(A, B)$, i.e., $z \in F_{A}(T)$. Therefore, $F_{A}(T)$ is convex. Similarly, it can be shown that $F_{B}(T)$ is a nonempty compact convex subset of $B_{0}$.

Lemma 29 Let $A, B$ be nonempty closed convex subsets of a global NPC space $X$ with the property that the closed convex hull of every finite subset of $X$ is compact. Let $A_{0}, B_{0}$ be nonempty compact convex and $T, S: A \cup B \rightarrow A \cup B$ be relatively u-continuous mappings such that $S$ and $T$ are commuting on $F_{A}(T) \cup F_{B}(T)$. Then $S\left(F_{A}(T)\right) \subseteq F_{B}(T)$ and $S\left(F_{B}(T)\right) \subseteq F_{A}(T)$.

Proof For each $x \in F_{A}(T)$, we have $d(x, T x)=\operatorname{dist}(A, B)$. Since $S$ is a relatively $u$-continuous mapping, then for $\delta>0$,

$$
d(x, T x)<\delta+\operatorname{dist}(A, B) \quad \text { implies } \quad d(S(x), S(T x))<\varepsilon+\operatorname{dist}(A, B)
$$


for each $\varepsilon>0$. Therefore, $d(S(x), S(T x))=\operatorname{dist}(A, B)$. The commutativity for $S$ and $T$ on $F_{A}(T)$ implies that $d(S(x), T(S x))=\operatorname{dist}(A, B)$. Thus, we deduce that $S x \in F_{B}(T)$. This shows that $S\left(F_{A}(T)\right) \subseteq F_{B}(T)$. Also, we can prove that $S\left(F_{B}(T)\right) \subseteq F_{A}(T)$.

Now, we define a new class of mappings called cyclic Banach pairs.

Definition 30 Let $A, B$ be nonempty subsets of a metric space $(X, d)$ and let $T, S: A \cup B \rightarrow$ $A \cup B$ be mappings. The pair $\{S, T\}$ is called a cyclic Banach pair if $S\left(F_{A}(T)\right) \subseteq F_{B}(T)$ and $S\left(F_{B}(T)\right) \subseteq F_{A}(T)$.

The following is an example of a pair of non-commuting mappings that are relatively $\mathrm{u}$-continuous and that are a cyclic Banach pair.

Example 31 Let $X=\mathbb{R}^{2}$ with the Euclidean metric and consider (as in [18])

$$
\begin{aligned}
& A=\left\{\left(0, \frac{1}{n}\right): n \in \mathbb{N}\right\} \cup\{(0,0)\}, \\
& B=\left\{\left(1, \frac{1}{n}\right): n \in \mathbb{N}\right\} \cup\{(1,0)\} .
\end{aligned}
$$

Let $T, S: A \cup B \rightarrow A \cup B$ be defined as

$$
\begin{aligned}
& T(0, x)=\left(1, \frac{x}{2}\right) \quad \text { for all }(0, x) \in A, \\
& T(1, x)=\left(0, \frac{x}{2}\right) \quad \text { for all }(1, x) \in B, \\
& S(0, x)=\left(1, \frac{x^{2}}{2}\right) \quad \text { for all }(0, x) \in A, \\
& S(1, x)=\left(0, \frac{x^{2}}{2}\right) \quad \text { for all }(1, x) \in B .
\end{aligned}
$$

Then $T$ and $S$ are relatively $u$-continuous mappings. Since

$$
T S(0, x) \neq S T(0, x),
$$

$T$ and $S$ are non-commuting mappings. Also, $\operatorname{dist}(A, B)=1$. It is easy to verify that

$$
F_{A}(T)=\{(0,0)\} \quad \text { and } \quad F_{B}(T)=\{(1,0)\}
$$

and

$$
S\left(F_{A}(T)\right) \subseteq F_{B}(T), \quad S\left(F_{B}(T)\right) \subseteq F_{A}(T) .
$$

Therefore, $\{S, T\}$ is a cyclic Banach pair.

The following theorem proves that two relatively $u$-continuous mappings which are not necessarily commuting have common best proximity points. 
Theorem 32 Let $A, B$ be nonempty closed convex subsets of a global NPC space $X$ with the property that the closed convex hull of every finite subset of $X$ is compact. Let $A_{0}, B_{0}$ be nonempty compact convex and $T, S: A \cup B \rightarrow A \cup B$ be affine relatively u-continuous mappings. If $\{S, T\}$ is a cyclic Banach pair, then $F_{A}(T) \cap F_{A}(S) \neq \emptyset$.

Proof By Theorem 28, $F_{A}(T)$ is a nonempty compact convex subset of $A_{0}$ and $F_{B}(T)$ is a nonempty compact convex subset of $B_{0}$. For each $x \in F_{A}(T)$, we have

$$
\operatorname{dist}(A, B) \leq \operatorname{dist}\left(F_{A}(T), F_{B}(T)\right) \leq d(x, T x)=\operatorname{dist}(A, B),
$$

which implies that $\operatorname{dist}\left(F_{A}(T), F_{B}(T)\right)=\operatorname{dist}(A, B)$. By the definition of cyclic Banach pairs $S: F_{A}(T) \cup F_{B}(T) \rightarrow F_{A}(T) \cup F_{B}(T)$. Since $\{S, T\}$ is a cyclic Banach pair and since for each $\varepsilon>0$ there exists a $\delta>0$ such that

$$
d(x, y)<\delta+\operatorname{dist}(A, B) \quad \text { implies } \quad d(S(x), S(y))<\varepsilon+\operatorname{dist}(A, B)
$$

for all $x \in F_{A}(T), y \in F_{B}(T)$, hence $S$ is a relatively $u$-continuous mapping on $F_{A}(T) \cup F_{B}(T)$. The conditions of Theorem 27 are satisfied, so there exists $x_{0} \in F_{A}(T)$ such that

$$
d\left(x_{0}, S x_{0}\right)=\operatorname{dist}\left(F_{A}(T), F_{B}(T)\right)=\operatorname{dist}(A, B) .
$$

Thus, $x_{0} \in F_{A}(S)$. This implies that $F_{A}(T) \cap F_{A}(S) \neq \emptyset$.

Next, we will extend Theorem 32 to the case of a countable family of not necessarily commuting relatively $u$-continuous mappings. Let $\Omega=\left\{T_{i}: i \in \mathbb{N}\right\}$ be a family of relatively $u$-continuous mappings. Define

$$
\begin{aligned}
& F_{A}\left(T_{i}\right)=\left\{x \in A: d\left(x, T_{i} x\right)=\operatorname{dist}(A, B)\right\}, \\
& F_{B}\left(T_{i}\right)=\left\{y \in B: d\left(y, T_{i} y\right)=\operatorname{dist}(A, B)\right\},
\end{aligned}
$$

for each $i=1, \ldots, n$.

Definition 33 Let $A, B$ be nonempty subsets of a metric space $(X, d)$ and let $T, S: A \cup B \rightarrow$ $A \cup B$. The pair $\{S, T\}$ is called a symmetric cyclic Banach pair if $\{S, T\}$ and $\{T, S\}$ are cyclic Banach pairs, that is, $S\left(F_{A}(T)\right) \subseteq F_{B}(T), S\left(F_{B}(T)\right) \subseteq F_{A}(T), T\left(F_{A}(S)\right) \subseteq F_{B}(S)$ and $T\left(F_{B}(S)\right) \subseteq F_{A}(S)$.

Theorem 34 Let $A, B$ be nonempty closed convex subsets of a global NPC space $X$ with the property that the closed convex hull of every finite subset of $X$ is compact. Let $A_{0}, B_{0}$ be nonempty compact convex and $\Omega$ a countable family of affine relatively u-continuous mappings such that $\left\{T_{i}, T_{j}\right\}$ is a symmetric cyclic Banach pair for each $i, j \in \mathbb{N}$. Then $\Omega$ has a common best proximity in $A$.

Proof First, we prove that $F_{A}\left(T_{1}\right) \cap F_{A}\left(T_{2}\right) \cap F_{A}\left(T_{3}\right) \neq \emptyset$. By an analogous argument to the proof of Theorem $32, F_{A}\left(T_{i}\right)$ is a nonempty compact convex subset of $A_{0}, F_{B}\left(T_{i}\right)$ is a 
nonempty compact convex subset of $B_{0}$ and $\operatorname{dist}\left(F_{A}\left(T_{i}\right), F_{B}\left(T_{i}\right)\right)=\operatorname{dist}(A, B)$, for $i=1,2,3$. So, we have $F_{A}\left(T_{1}\right) \cap F_{A}\left(T_{2}\right)$ and $F_{B}\left(T_{1}\right) \cap F_{B}\left(T_{2}\right)$ are nonempty compact convex with

$$
\operatorname{dist}\left(F_{A}\left(T_{1}\right) \cap F_{A}\left(T_{2}\right), F_{B}\left(T_{1}\right) \cap F_{B}\left(T_{2}\right)\right)=\operatorname{dist}(A, B) .
$$

Suppose that $T_{3}$ is a mapping on $\left(F_{A}\left(T_{1}\right) \cap F_{A}\left(T_{2}\right)\right) \cup\left(F_{B}\left(T_{1}\right) \cap F_{B}\left(T_{2}\right)\right)$. Since both of $\left\{T_{3}, T_{1}\right\}$ and $\left\{T_{3}, T_{2}\right\}$ are cyclic Banach pairs, $T_{3}$ is a relatively $u$-continuous mapping on $\left(F_{A}\left(T_{1}\right) \cap F_{A}\left(T_{2}\right)\right) \cup\left(F_{B}\left(T_{1}\right) \cap F_{B}\left(T_{2}\right)\right)$. From Theorem $27, T_{3}$ has a best proximity point $z \in F_{A}\left(T_{1}\right) \cap F_{A}\left(T_{2}\right)$. This shows that $F_{A}\left(T_{1}\right) \cap F_{A}\left(T_{2}\right) \cap F_{A}\left(T_{3}\right) \neq \emptyset$.

By induction, for a finite symmetric cyclic Banach family $\Omega^{\prime}=\left\{T_{1}, T_{2}, \ldots, T_{n}\right\}$ of affine relatively $u$-continuous mappings, there exists $x_{0} \in \bigcap_{i=1}^{n} F_{A}\left(T_{i}\right)$.

Now, let $\Omega=\left\{T_{i}: i \in \mathbb{N}\right\}$. For each $T_{i}, F_{A}\left(T_{i}\right)$ is a nonempty compact convex of $A_{0}$, and for $i=1, \ldots, n$, we have

$$
\bigcap_{i=1}^{n} F_{A}\left(T_{i}\right) \neq \emptyset .
$$

This shows that the set $\left\{F_{A}\left(T_{i}\right): i \in \mathbb{N}\right\}$ has a finite intersection property. Thus, we have

$$
\bigcap_{i=1}^{\infty} F_{A}\left(T_{i}\right) \neq \emptyset
$$

i.e., $\Gamma$ has a common best proximity point in $A$.

\section{Competing interests}

The authors declare that they have no competing interests.

\section{Authors' contributions}

All authors contributed equally and significantly in writing this article. All authors read and approved the final manuscript.

\section{Author details}

'Department of Mathematics, Science Faculty for Girls, King Abdulaziz University, P.O. Box 4087, Jeddah, 21491, Saudi Arabia. ${ }^{2}$ Department of Mathematics, King Abdulaziz University, P.O. Box 80203, Jeddah, 21859, Saudi Arabia.

\section{Acknowledgements}

This work was funded by the Deanship of Scientific Research (DSR), King Abdulaziz University, Jeddah, under grant No. (3-843-D1432). The authors, therefore, acknowledge DSR with thanks for technical and financial support. The authors are in debt to the anonymous reviewers whose comments helped improve the quality of the paper.

Received: 17 May 2012 Accepted: 7 November 2012 Published: 28 December 2012

\section{References}

1. Fan, K: Extensions of two fixed point theorems of F. E. Browder. Math. Z. 122, 234-240 (1969)

2. Eldred, AA, Kirk, WA, Veeramani, P: Proximal normal structure and relatively nonexpansive mappings. Stud. Math. 171(3), 283-293 (2005)

3. Eldred, AA, Veeramani, P: Existence and convergence of best proximity points. J. Math. Anal. Appl. 323(2), 1001-1006 (2006)

4. Anuradha, J, Veeramani, P: Proximal pointwise contraction. Topol. Appl. 156(18), 2942-2948 (2009)

5. Al-Thagafi, MA, Shahzad, N: Convergence and existence results for best proximity points. Nonlinear Anal. 70, 3665-3671 (2009)

6. Markin, J, Shahzad, N: Best approximation theorems for nonexpansive and condensing mappings in hyperconvex spaces. Nonlinear Anal. 70(6), 2435-2441 (2009)

7. Vetro, C: Best proximity points: convergence and existence theorems for - cyclic mappings. Nonlinear Anal. 73 2283-2291 (2010)

8. Sankar Raj, V, Veeramani, P: Best proximity pair theorems for relatively nonexpansive mappings. Appl. Gen. Topol. 10(1), 21-28 (2009) 
9. Eldred, AA, Sankar Raj, V, Veeramani, P: On best proximity pair theorems for relatively u-continuous mappings. Nonlinear Anal. 74, 3870-3875 (2011)

10. Fernández-León, A: Existence and uniqueness of best proximity points in geodesic metric spaces. Nonlinear Anal. 73, 915-921 (2010)

11. Bridson, MR, Haefliger, A: Metric Spaces of Non-Positive Curvature. Springer, Berlin (1999)

12. Papadopoulus, A: Metric Spaces, Convexity and Nonpositive Curvature. European Math. Soc., Zurich (2005)

13. Sturm, KT: Probability measures on metric spaces of nonpositive curvature. In: Auscher, P, Coulhon, T, Grigor'yan, A (eds.) Heat Kernels and Analysis on Manifolds, Graphs, and Metric Spaces. Lecture Notes from a Quarter Program on Heat Kernels, Random Walks, and Analysis on Manifolds and Graphs. Paris, France. Contemporary Mathematics, vol. 338, pp. 357-390 (2003)

14. Ballmann, W: Lectures in Spaces of Nonpositive Curvature. DMV Seminar, vol. 25. Birkhäuser, Basel (2005)

15. Jost, J: Nonpositive Curvature: Geometric and Analytic Aspects. Lectures in Mathematics ETH Zurich. Birkhäuser, Basel (1997)

16. Niculescu, CP, Roventa, I: Fan's inequality in geodesic spaces. Appl. Math. Lett. 22, 1529-1533 (2009)

17. Niculescu, CP, Roventa, I: Schauder fixed point theorem in spaces with global nonpositive curvature. Fixed Point Theory Appl. 2009, Article ID 906727 (2009)

18. Sankar Raj, V: A best proximity point theorem for weakly contractive non-self-mappings. Nonlinear Anal. 74 4804-4808 (2011)

doi:10.1186/1687-1812-2012-234

Cite this article as: Alghamdi et al.: Best proximity point results in geodesic metric spaces. Fixed Point Theory and Applications 2012 2012:234.

\section{Submit your manuscript to a SpringerOpen ${ }^{\ominus}$ journal and benefit from:}

- Convenient online submission

- Rigorous peer review

- Immediate publication on acceptance

Open access: articles freely available online

- High visibility within the field

- Retaining the copyright to your article 